\title{
A SARS Postcard - Reflections of Two Healthcare Leaders
}

\author{
Lynn M. Nagle, RN, PhD \\ Chief Information Officer, Mount Sinai Hospital, Toronto \\ Leslie Vincent, RN, MScA, $\operatorname{CON}(\mathrm{C})$ \\ Vice President, Nursing, Mount Sinai Hospital, Toronto
}

Summarizing our reflections on the past eight weeks is a daunting task. The opportunity to provide leadership for a hospital that has met the challenge of caring for clients with SARS (severe acute respiratory syndrome) has been unique. It has given us new understanding of system-wide and organizational processes, crisis management, our staff, the true meaning of organizational culture and teamwork and our own capacity to sustain leadership in the face of uncertainty.

The SARS outbreak unleashed an unprecedented demand for leadership, courage and resilience among healthcare providers. The discovery of a new, highly contagious illness, with an almost $20 \%$ mortality rate, thrust the Ontario healthcare system into an emergency unparallelled in recent times. Although our experience and that of other healthcare administrators is likely quite different from that of the frontline staff, it is rich with lessons learned that can be applied in the future. Indeed, our own personal involvement included home quarantine when one of our colleagues had been diagnosed as a suspected SARS case.

It is difficult to explain the impact of these events to others who have not been as closely involved with the Toronto SARS crisis. When we shared our observation that this would change healthcare forever, we were often asked why. Between us, we have about 54 years of healthcare experience, yet neither of us has ever before witnessed such an enduring test of leadership. Creating an air of calm, competence, trust, transparency and confidence was probably the single most important element of managing this event. We have shared the stress and emotional upset of conveying messages that we knew would raise great anxiety. There is no more difficult task than to share the news that members of your own staff have been 
diagnosed with SARS and are being cared for in your own hospital, or to see a member of the nursing staff vilified in the media just for doing her job and, unfortunately, developing SARS. These were emotionally demanding circumstances for us all.

Beyond the responsibility of caring for people with SARS, there was the continuing challenge of providing care to thousands of clients with other healthcare needs. From the moment that a citywide Code Orange was declared, the mobilization of resources in hospitals and the community has been unprecedented.

Managing concerns about the adequacy of protective supplies, the creation of a dedicated SARS unit, the introduction and monitoring of new isolation procedures almost daily, the establishment of extensive screening for all staff and clients prior to entry to the hospital - none of these were trivial tasks. The turmoil of crisis management invaded our lives. The daily challenges of keeping pace with constantly changing directives from the Provincial Operations Centre and the Ministry of Health were sufficient to keep at least a dozen administrators consumed. Extreme demands were placed on the Infection Control, Microbiology and Occupational Health Departments, requiring additional nurses to support these services around the clock. Early on, incidents of unprotected exposure to SARS in the ICU and ER resulted in nurses and other staff being sent home in quarantine, necessitating the redeployment of staff from other nursing units to fill in the gaps. Mandatory screening at entrance points required the reassignment of dozens of staff every day. Many individuals throughout the organization were asked to assume new and unfamiliar responsibilities.

As the two senior administrators leading the hospital during this crisis, we relied upon the expertise and commitment of a core team of senior staff. The initial long days of crisis management rapidly evolved into a routine of twice-daily senior management meetings and the formation of task groups for new functions: infection control procedures, supply logistics, screening, clinical services decision-making, communications and so forth. A clear, authoritative leadership style helped maintain focus on priorities, rapid decision-making, formation of task groups, alignment of staff with new procedures and expectations, effective execution of new directives and ongoing communication. Maintaining a personal demeanour of control and calm reason supported others in their roles. The seeking and accepting of personal support was crucial, and we will always be grateful to our colleagues and families.

Information and communication mechanisms were quickly established both within the organization and across the Greater Toronto Area. Mount Sinai Hospital became part of the newly established virtual West Network that comprised all hospitals on the west side of Toronto, extending to the Hamilton border. Daily conference calls for the Network provided a forum for sharing of hospital status 
reports, problem-solving, and collaborative decision-making.

The overall impacts of this outbreak have yet to be measured. Within healthcare alone, the physical and emotional costs have been enormous: to the system, to clients and families and to healthcare workers. In Toronto, the stigma of SARS has taken an unknown toll on the business community.

In looking for the positives, we have found many. Healthcare workers have been united in unprecedented ways. In our organization, the evidence of individual and collective commitment to do what was needed is overwhelming.
We are proud to be part of a team that so profoundly cared about managing this situation for the sake of all concerned. Despite our collective physical and psychological fatigue, we have succeeded in overcoming and managing the challenges of the SARS outbreak. Our story is one of many in Toronto and in Asia. Our colleagues in the many organizations that met this crisis deserve our respect. In a future issue, we will chronicle our experience in more detail, but until then, take it from us - nurse leaders matter. And while it has been our privilege to be leaders, our staff are the heroes!

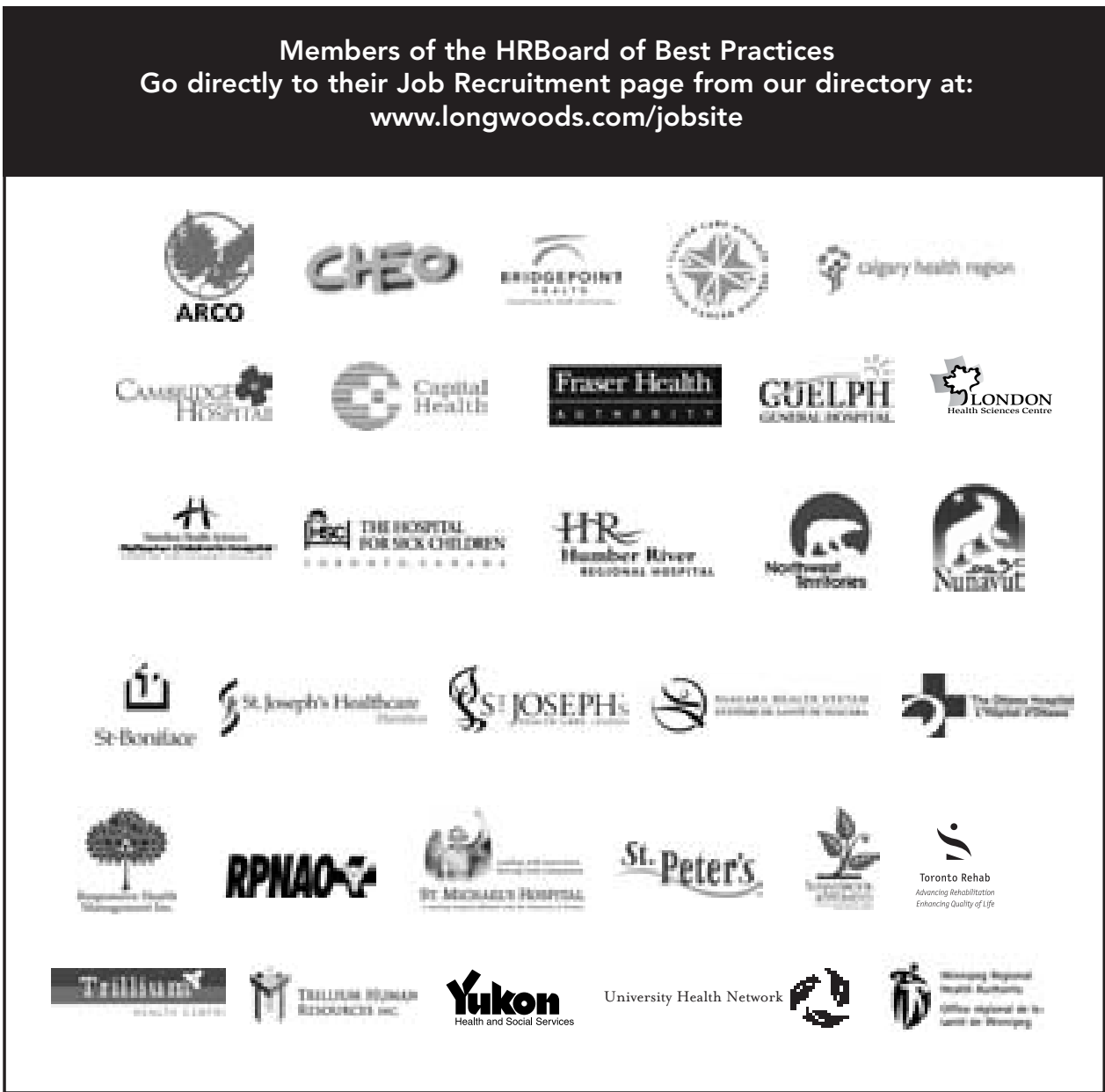

